\title{
Relationship between Solubility of Chitosan in Alcoholic Solution and Its Gelation
}

\author{
Motohiko Sano, ${ }^{a}$ Osamu Hosoya, ${ }^{b}$ Shose TaOka, ${ }^{a}$ Toshinobu Seki,${ }^{a, b}$ Takeo Kawaguchi, ${ }^{a}$ \\ Kenji Sugibayashi, ${ }^{a, b}$ Kazuhiko Juni, ${ }^{a}$ and Yasunori Morimoto ${ }^{*, a, b}$ \\ Faculty of Pharmaceutical Sciences, Josai University,"1-1 Keyakidai, Sakado, Saitama 350-0295, Japan and Research \\ Institute of TTS Technology, ${ }^{b}$ 1-1 Keyakidai, Sakado, Saitama 350-0295, Japan. \\ Received February 25, 1999; accepted April 13, 1999
}

\begin{abstract}
A cationic polymer, chitosan $(\mathrm{CHI})$ is anticipated to be a potent component in several dosage forms involving transdermal drug delivery systems (TDDS). Solubility of CHI and its gelation in alcoholic aqueous solutions were investigated. Elasticity of the gel containing $3 \% \mathrm{CHI}, 2 \%$ oxalic acid, as a crosslinking agent, and ethanol (EtOH) and/or 1,3-butyleneglycol (BG) was also evaluated by a rheometer. The CHI solubility in the gel was dependent on the solubility parameters of the mixed solvent. High elasticity was observed in the CHI gel when an alcoholic solvent giving low solubility of $\mathrm{CHI}$ was used. A good correlation was found between the gel elasticity and the relative gel concentration (=concentration of $\mathrm{CHI}$ in gel/solubility of $\mathrm{CHI}$ in gel). Since the polarity of mixed solvents also affected the thermodynamic activities of drugs and penetration enhancers, optimization of the vehicle composition for TDDS is important to assure suitable drug efficacy.
\end{abstract}

Key words chitosan; gelation; ethanol; 1,3-butyleneglycol; solubility parameter

Several polymer materials have been utilized in a number of pharmaceutical preparations and potential drug delivery systems (DDS). ${ }^{1,2}$ Selection and/or use of polymers are very important in the formulation design, since a polymer-drug interaction is one of the major determinants of the quality of a pharmaceutical preparation. On the other hand, aqueous solutions containing ethanol $(\mathrm{EtOH})$ and other alcohol are thought to be a practical chemical enhancer to increase permeation of drugs through biological membrane because of their safety and strong enhancing effect. ${ }^{3,4)} \mathrm{EtOH}$ has already been used in several marketed transdermal DDS (TDDS). Most of them are a liquid reservoir type of TDDS, in which active drugs and penetration enhancers are dissolved in reservoir solvents and are sealed by drug-permeable membranes. $^{5-7)}$ With the liquid reservoir type of TDDS it is difficult to maintain an effective application area because of change of patient position and there is a high risk of breaking the membranes. Selection of a highly viscous gel for the reservoir medium may relieve these problems.

We have reported that the skin permeation of morphine was enhanced by a mixed solvent system of $l$-menthol, EtOH and water, ${ }^{4}$ and the enhancing effect was influenced by several polymers. ${ }^{8)}$ The latter study ${ }^{8)}$ indicated that the skin permeation of morphine as a basic compound was increased by chitosan $(\mathrm{CHI})$ as a cationic polysaccharide, while it was decreased by carboxyvinylpolymer as an anionic polymer.

In the present study, we investigated the solubility and gelation of $\mathrm{CHI}$ in aqueous solutions containing alcohols in detail to obtain further information on $\mathrm{CHI}$ as a new pharmaceutical additive suitable for TDDS.

\section{Experimental}

Materials CHI was supplied by Ajinomoto Co., Inc. (Marine Dew PC100, M.W. 450000-900000, Tokyo, Japan). The CHI has a degree of deacetylation of about $50 \%$. EtOH, isopropanol, propyleneglycol and glycerol were purchased from Wako Pure Chemical Industries Co., Ltd. (Osaka, Japan). 1,3-Butylene glycol (BG) was purchased from Tokyo Chemical Industries Co., Ltd. (Tokyo). Other chemicals were of reagent grade.

Evaluation of Effect of Alcohols on the CHI Solubility Different ratios of alcohol and purified water were mixed in a transparent glass vial, and

* To whom correspondence should be addressed.
$\mathrm{CHI}$ was added at concentration of $3 \%$ to the mixed solvent. Since $\mathrm{CHI}$ is insoluble in neutral solutions, acetic acid was added at concentration of $2 \%$ to each vial. CHI was dispersed and dissolved by stirring with a vortex mixer and ultrasonication. The obtained solution was stored at $32^{\circ} \mathrm{C}$ for $48 \mathrm{~h}$ in an incubator, and uniformity of $\mathrm{CHI}$ in the solution was judged visually. The maximum concentration of alcohol which allowed complete dissolution of $\mathrm{CHI}$ in the solution was called the "critical concentration."

Preparation of CHI Gel and Evaluation of $\mathrm{CHI}$ Solubility in the Gel Various amounts of $\mathrm{CHI}$ and $2 \%$ oxalic acid as a crosslinking agent were added to several mixed solutions of $\mathrm{EtOH}, \mathrm{BG}$ and water in a transparent glass vial. They were mixed rapidly to obtain a clear solution or gel as above, and the resulting solutions or gels were stored at $32^{\circ} \mathrm{C}$ for $48 \mathrm{~h}$. The transparency of the gel was optically checked. The maximum concentration of CHI which retained the uniformity and transparency of the gel was regarded as "CHI solubility".

Evaluation of CHI Gelation Stress relaxation of CHI gel was measured by a rheometer (NRM-2002J, Fudo Kogyo Co., Ltd. Tokyo), which had a circular flat plunger (diameter $20 \mathrm{~mm}$, thickness $1 \mathrm{~mm}$ ) jointed to a sensor and a rising sample stand. Since the same strain was not achieved for each $\mathrm{CHI}$ gel, the stress relaxation was examined at four different constant strains. The CHI gel in a glass vial (volume $50 \mathrm{ml}$, internal diameter $32 \mathrm{~mm}$, height $75 \mathrm{~mm}$ ) rose at the rate of $30 \mathrm{~mm} / \mathrm{min}$ and stopped at a strain in the range of 0.02 to 0.2 . Modulus of elasticity of the $\mathrm{CHI}$ gel was calculated at each point using the following equation:

$$
E=\frac{F \cdot L}{a \cdot l}
$$

where $E$ is modulus elasticity, $F$ and $L$ represent stress and initial length of the CHI gel, respectively, and $a$ and $l$ are area of the plunger and compressed length of the $\mathrm{CHI}$ gel. The maximum and stationary moduli, $E_{0}$ and $E_{\infty}$, of elasticity were obtained from the time course of $E . E_{\mathrm{o}}$ and $E_{\mathrm{x}} / E_{\mathrm{o}}$ at a strain of 0.04 , which were obtained by a regression equation of the moduli of elasticity against the strains, were used for indices of gel strength and maintenance of shape, respectively. For instance, $E_{x} / E_{0}=1$ was expressed as a complete elastic body.

\section{Results and Discussion}

Effect of Alcohols on CHI Solubility The influence of different polarities of alcohols added to the aqueous solution on the $\mathrm{CHI}$ solubility was evaluated. Figure 1 shows the critical concentration of alcohols to retain a condition in which $3 \% \mathrm{CHI}$ was clearly dissolved. The alcohols are arranged in the order of polarity in Fig. $1 .^{97}$ The highest critical concen-

(C) 1999 Pharmaceutical Society of Japan 
tration was observed for glycerol, which has the highest polarity among the alcohols tested. The result suggested that solubility of $\mathrm{CHI}$ could be affected by the polarity of the mixed solvent.

Aqueous solutions containing $\mathrm{EtOH}$ and/or BG were used for the gel preparation to evaluate the effect of polarity of the mixed solvent system on the $\mathrm{CHI}$ solubility in the $\mathrm{CHI}$ gel. ${ }^{5,10)}$ Figure 2A shows $\mathrm{CHI}$ solubility in the gel containing $\mathrm{EtOH}$ and water. Solubility of $\mathrm{CHI}$ in water was $10 \%$, which was the maximum value in this series. CHI solubility decreased almost linearly with the EtOH content, and in $50 \%$ EtOH was less than $0.1 \%$. Figures $2 \mathrm{~B}$ and $2 \mathrm{C}$ show $\mathrm{CHI}$ solubility in the gel containing EtOH, BG and water; the solubility increased with increase in $\mathrm{BG} / \mathrm{EtOH}$ ratio. Since the polarity of BG is higher than that of EtOH, hydrophilicity of the mixed solvent increases with increase in the ratio.

The solubility parameter (SP) of each mixed solvent was calculated to determine the relationship between solvent polarity and the $\mathrm{CHI}$ solubility. ${ }^{11)}$ Figure 3 illustrates the $\mathrm{CHI}$ solubility in each mixed solution against corresponding SP value; the solubility in the gel had a tendency to increase with the SP value. The SP value of $\mathrm{CHI}$ itself seemed to be

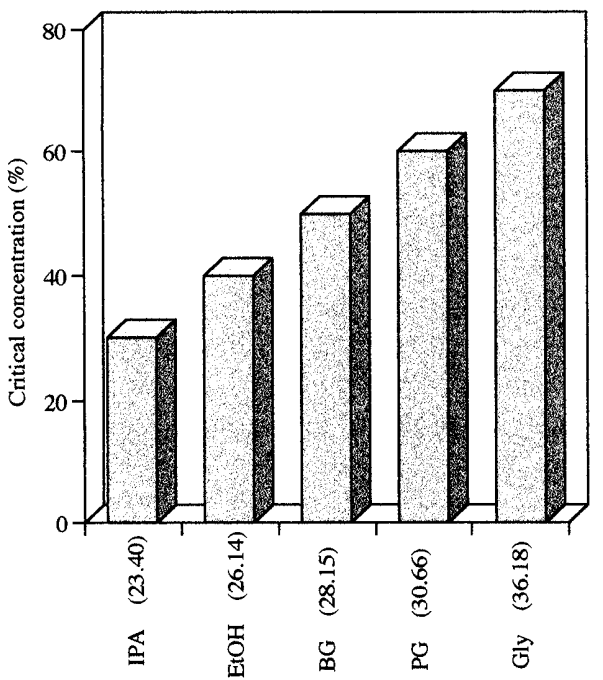

Fig. 1. Critical Concentration of Alcohol in 3\% CHI Solution Containing $2 \%$ Acetic Acid

IPA, isopropanol; PG, propyleneglycol; Gly, glycerol: (SP of each alcohol in parentheses, $\left.\left(\mathrm{J} / \mathrm{cm}^{3}\right)^{1 / 2}\right)$. higher than that of the mixed solvent system. The CHI solubility in highly polar solvents could be high because of low dissolution heat and high solvation of amino group or hydroxyl group, as true of the dissolution behavior of polymer described by Flory. ${ }^{12)}$

Effect of Alcohols on Gelation of CHI The viscoelastic properties of the CHI gel were measured to learn the effect of alcohols on the gelation of CHI. Figure 4 shows $E_{\mathrm{o}}$ and $E_{x} / E_{\mathrm{o}}$ at several compositions of the gel in which $E_{0}$ is the maximum elasticity and $E_{x} / E_{0}$ is maintenance of its shape. These values in the EtOH-water mixed solvent system containing $3 \% \mathrm{CHI}$ and $2 \%$ oxalic acid varied with the $\mathrm{EtOH}$ content in the gel (Fig. 4A). At $40 \% \mathrm{EtOH}, E_{\mathrm{o}}$ and $E_{x} / E_{\mathrm{o}}$ were $9.4 \times 10^{3}$ $\left(\mathrm{N} / \mathrm{m}^{2}\right)$ and 0.64 , respectively. Both values were maximum in this series. For the gel containing $50 \%$ EtOH, phase-separation was observed, i.e., $\mathrm{CHI}$ was deposited, and the values of $E_{\mathrm{o}}$ and $E_{\infty} / E_{\mathrm{o}}$ were lower than those of $40 \% \mathrm{EtOH}$. On the other hand, the gel containing $50 \% \mathrm{BG}$ was uniform and the values of $E_{\mathrm{o}}$ and $E_{\infty} / E_{\mathrm{o}}$ were higher than those of $50 \% \mathrm{EtOH}$ (Fig. 4C). In the gel containing both EtOH and BG (Fig. 4B, C), the values of $E_{\mathrm{o}}$ and $E_{\infty} / E_{\mathrm{o}}$ decreased with an increase in the $\mathrm{BG} / \mathrm{EtOH}$ ratio, and the highest values were observed in the gel containing $40 \% \mathrm{EtOH}$ and $10 \% \mathrm{BG}$. Substitution of only $10 \%$ in $50 \%$ EtOH by BG led to a significant change in the elasticity of $\mathrm{CHI}$ gel. These results suggest that the gel elasticity depends on the polarity of the mixed solvent rather

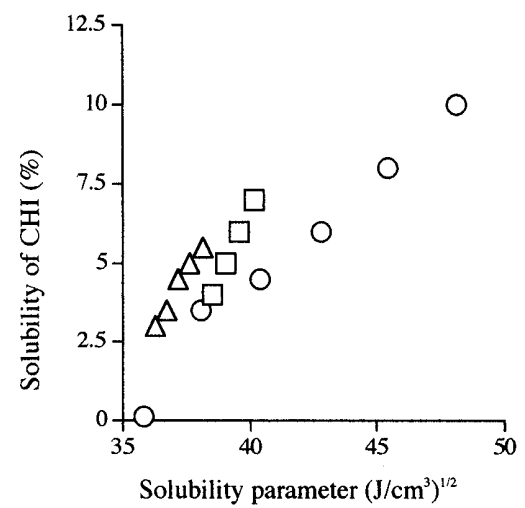

Fig. 3. Relationship between SP of Mixed Solvent and Solubility of CHI in the Gel Containing 2\% Oxalic Acid

Data in Fig. 2 were re-plotted as the relationship between SP and solubility.

O, 0-50\% EtOH/water (Fig. $2 \mathrm{~A}$ ); $\square, \mathrm{EtOH}+\mathrm{BG}$ (total 40\%)/water (Fig. 2 B); $\triangle, \mathrm{EtOH}+\mathrm{BG}$ (total $50 \%$ )/water (Fig. $2 \mathrm{C}$ ).
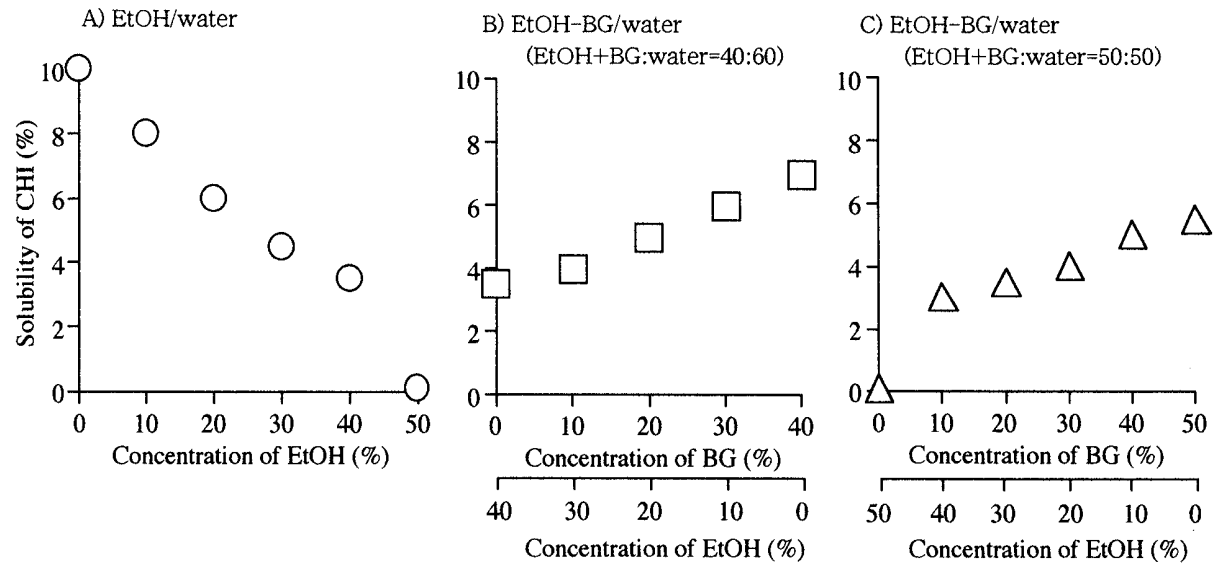

Fig. 2. Solubility of $\mathrm{CHI}$ in the $\mathrm{CHI}$ Gel Containing Alcohols and $2 \%$ Oxalic Acid

A) $0-50 \% \mathrm{EtOH} /$ water; B) $\mathrm{EtOH}+\mathrm{BG}$ (total $40 \%$ ) $/$ water; C) EtOH+BG (total $50 \%$ ) $/$ water. 
A) $\mathrm{EtOH} /$ water

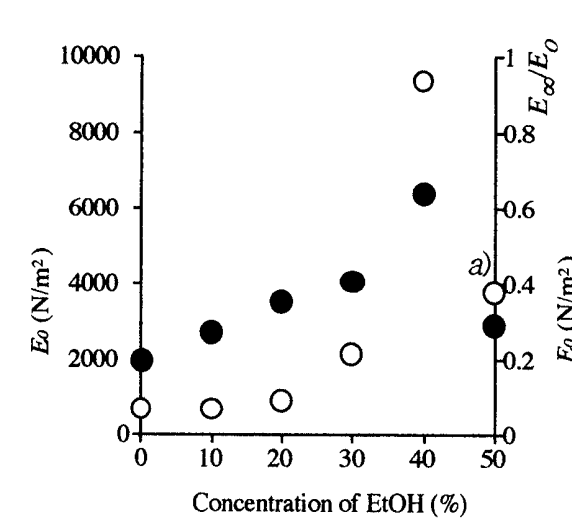

B) $\mathrm{EtOH}-\mathrm{BG} /$ water

$(\mathrm{EtOH}+\mathrm{BG}:$ water $=40: 60)$
C) $\mathrm{EtOH}-\mathrm{BG} /$ water

(EtOH+BG:water=50:50)
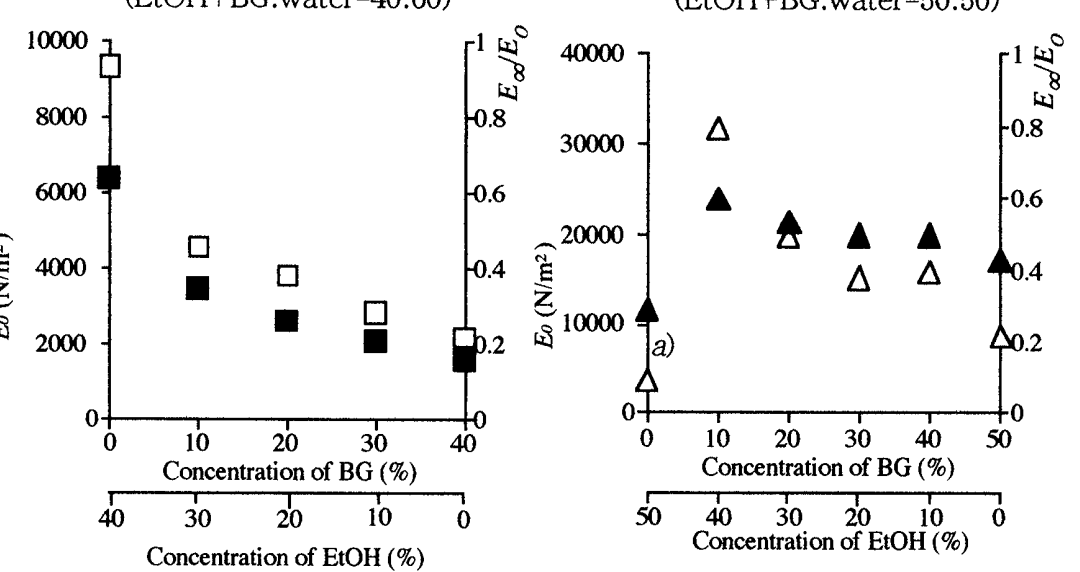

Fig. 4. $E_{0}$ and $E_{x} / E_{0}$ of $3 \%$ CHI Gels Containing Alcohols and $2 \%$ Oxalic Acid

A) $0-50 \% \mathrm{EtOH} /$ water; B) $\mathrm{EtOH}+\mathrm{BG}($ total $40 \%) /$ water; C) EtOH+BG (total $50 \%) /$ water: $E_{0}(O, \square, \triangle), E_{x} / E_{0}$
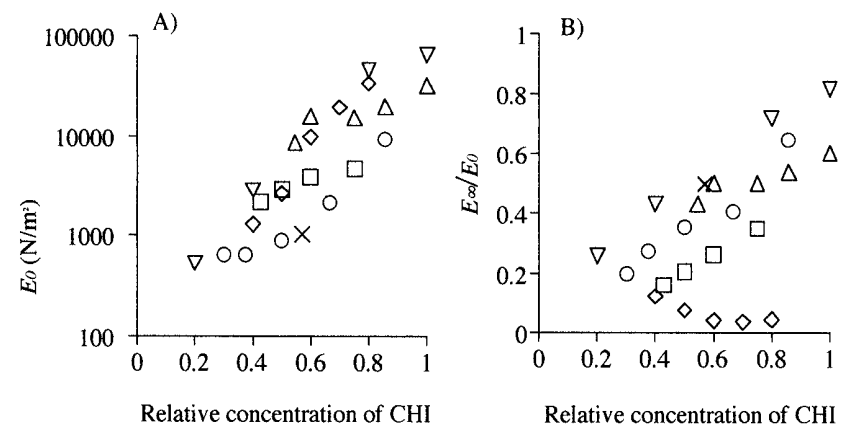

Fig. 5. Relationship between $E_{0}$ and $E_{x} / E_{0}$ and Relative Concentration of $\mathrm{CHI}$ (Concentration / Solubility of $\mathrm{CHI}$ in the Gel Containing $2 \%$ Oxalic Acid)

O, 3\% CHI, 0-50\% EtOH/water; $\square, 3 \% \mathrm{CHI}$, EtOH+BG (total $40 \%$ )/water; $\triangle, 3 \%$ $\mathrm{CHI}$, EtOH+BG (total $50 \%$ ) $/$ water; $\diamond, 4-8 \% \mathrm{CHI} /$ water; $\nabla, 1-5 \% \mathrm{CHI}, 20 \% \mathrm{EtOH}$ $30 \% \mathrm{BG} /$ water; $\times, 2 \% \mathrm{CHI}, 40 \% \mathrm{EtOH} /$ water.

than on the chemical structure of solvents. CHI dissolved in a solvent in which it is poorly soluble, could effectively act for the gelation. The kind and content of alcohols are important in preparation of a $\mathrm{CHI}$ gel in which the alcohols act as skin penetration enhancers for TDDS.

High elasticity was observed in the $\mathrm{CHI}$ gel, which was uniform and consisted of an alcoholic solution in which CHI had low solubility. Relative concentrations of $\mathrm{CHI}$ in several alcoholic gels were calculated to examine the relationship between CHI solubility in the alcoholic solution and elasticity of the gel as follows:

relative concentration $=($ concentration of $\mathrm{CHI} /$ solubility of $\mathrm{CHI})$

$E_{\mathrm{o}}$ and $E_{\infty} / E_{\mathrm{o}}$ were plotted against these values. $E_{\mathrm{o}}$ and $E_{\infty} / E_{\mathrm{o}}$ values of $\mathrm{CHI}$ gel containing various concentrations of $\mathrm{CHI}$ $(1-8 \%)$ were added to the data in Fig. 4, which was observed in the $\mathrm{CHI}$ gel containing $3 \% \mathrm{CHI}$. Figure $5 \mathrm{~A}$ shows a semi-logarithmic plot of $E_{\mathrm{o}}$ against the relative concentration of $\mathrm{CHI}$, suggesting a positive correlation. Figure $5 \mathrm{~B}$ shows a linear plot of $E_{x} / E_{\mathrm{o}}$ against the relative concentration of $\mathrm{CHI}$; it suggests a positive correlation between solubility of $\mathrm{CHI}$ and elasticity of its gel, except in the case of $\mathrm{CHI}$ gel without alcohols. The exceptional behavior observed in the system without alcohols is not fully understood, and further study to clarify possible differences in gelling behavior of $\mathrm{CHI}$ in aqueous systems with and without alcohols is needed.

\section{Conclusion}

This study examined the relationship between CHI solubility in alcoholic solution and its gelation. The results suggest that the polarity of mixed solvents affects not only the solubility of CHI but also elasticity of the gel. Since the polarity of mixed solvents also affects the thermodynamic activity of drugs and penetration enhancers, such as 1 -menthol, ${ }^{13)}$ it is clear that optimization of the composition of vehicle for TDDS is important.

\section{References}

1) Kydonieus A. (ed.), "Treatise on Controlled Drug Delivery," Marcel Dekker, New York, 1992.

2) Sugibayashi K., Morimoto Y., J. Contr. Rel., 29, 177-185 (1994).

3) Sugibayashi K., Kobayashi D., Nakagaki E., Hatanaka T., Inoue N., Kusumi S., Kobayashi M., Kimura M., Morimoto Y., Int. J. Pharmaceut., 113, 189-197 (1995).

4) Tsukagoshi S., Horikoshi N., Takahashi S., Kato C., Shimizu Y., Suminaga M., Kitajima T., Seki T., Morimoto Y., Drug Delivery System, 11, 393-397 (1996).

5) Pershing L. K., Lambert L. D., Knutson K., Pharm. Res., 7, 170-175 (1990).

6) Campbell P. S., Chandrasekaran S. K., U. S. Patent 4379454 (1983).

7) Gale R. M., Lee E. S., Taskovich L. T., Yum S., U. S. Patent 4588580 (1986).

8) Hosoya O., Sano M., Wada Y., Seki T., Sugibayashi K., Juni K., Morimoto Y., Chem. Pharm. Bull., 46, 882-885 (1998).

9) Hoy K. L., J. Paint Technol., 42, 76-118 (1970).

10) Matsuzawa T., Wada Y., Shimoyama M., Nakajima K., Seki T., Sugibayashi K., Morimoto Y., Biopharm. Drug Disp., 15, 665-678 (1994).

11) Adjei A., Newburger J., Martin A., J. Pharm. Sci., 69, 659-661 (1980).

12) Flory P. J., "Principles of Polymer Chemistry," Cornell University, Ithaca, NY, 1953.

13) Wada Y., Nakajima K., Yamazaki J., Seki T., Sugibayashi K., Morimoto Y., Biol. Pharm. Bull., 16, 600-603 (1993). 